\title{
Location-specific versus hemisphere-specific adaptation of processing selectivity
}

\author{
Mike Wendt, Rainer H. Kluwe, and Ina Vietze \\ Helmut Schmidt University, Hamburg, Germany \\ and University of the Federal Armed Forces, Hamburg, Germany
}

\begin{abstract}
Current theories of cognitive control assume that processing selectivity is adjusted according to the utility of processing task-irrelevant stimulus features. Consistently, interference evoked by flanker stimuli is reduced when the proportion of incompatible trials - in which flankers are associated with an incorrect response-is increased. Consistent with the idea that the cerebral hemispheres select processing strategies independently of each other, Corballis and Gratton (2003) demonstrated that flanker interference for stimuli presented in either the left or right visual hemifield is affected by the ratio of compatible and incompatible target-flanker pairings presented in the same - but not in the other-hemifield. Presenting stimuli at four different locations, we demonstrated independent effects of the ratio of compatible and incompatible trials for stimulus locations in different hemifields as well as for stimulus locations within the same hemifield. Independent selectivity adjustment regarding the left and right visual hemifields thus appears to be a special case of a larger class of location-specific adaptation effects and might not be informative regarding hemisphere-specific processing.
\end{abstract}

Interference paradigms demonstrate that when people have to respond to a specific stimulus feature, processing selectivity is often incomplete in the sense that additional stimulus features - which are logically not needed for successful task performance - are nevertheless processed up to a level at which they affect performance. This phenomenon is indicated most prominently by interference effects - that is, slower and less accurate performance on trials on which an irrelevant stimulus feature is associated with a response different from the one needed for correct performance (henceforth termed incompatible) than on trials on which both relevant and irrelevant stimulus features are associated with the same response (henceforth termed compatible). For instance, in the Eriksen flanker task (Eriksen \& Eriksen, 1974), participants are asked to classify a central target stimulus - for example, the letter $H$ versus the letter $S$ - that is embedded in a number of adjacent letters (flankers) by pressing a corresponding response key. Performance is typically worse on incompatible trials (e.g., $H H S H H$ ) than on compatible trials (e.g., SSSSS).

Current models of cognitive control assume that the human processing system is sensitive to the utility of processing irrelevant stimulus information-for instance, whether processing a flanker in the Eriksen task activates a correct or an incorrect response. These models also assume that the human processing system responds by optimizing the processing strategy - that is, by adjusting the weights given to processing task-relevant and taskirrelevant stimulus features (see, e.g., Botvinick, Braver,
Barch, Carter, \& Cohen, 2001; Gratton, Coles, \& Donchin, 1992). Consistent with this idea, reductions of interference evoked by processing irrelevant stimulus features under conditions of increased frequency of incompatible trials and/or reduced frequency of compatible trials has been found in various interference paradigms, including the flanker task (Corballis \& Gratton, 2003; Gratton et al., 1992), different variants of the Stroop task (Logan \& Zbrodoff, 1979; Tzelgov, Henik, \& Berger, 1992), and the Simon task (Hommel, 1994; Stürmer, Leuthold, Soetens, Schröter, \& Sommer, 2002). That is, less weight seems to be given to the processing of an irrelevant stimulus dimension (such as a word in the Stroop task, characters adjacent to the target stimulus in the flanker task, or the stimulus location in the Simon task), if the processing of that dimension is more likely to impair performance.

Recent research suggests that the human cognitive system is capable of rapidly switching between different degrees of selectivity (Crump, Gong, \& Milliken, 2006; Jacoby, Lindsay, \& Hessels, 2003) or perhaps even of maintaining two different degrees of selectivity at the same time (Corballis \& Gratton, 2003). Of most importance to the present study, Corballis and Gratton examined the role of the cerebral hemispheres in adjustments of visuospatial selectivity. To this end, a flanker task was administered in which stimuli were presented randomly at a location in either the left or the right visual hemifield. Manipulating the ratios of compatible and incompatible trials at the two locations independently of each other, Corballis and Gratton found enhancement of flanker in-

M.Wendt, mike.wendt@hsu-hh.de 
terference in a given visual hemifield when the proportion of compatible trials in that hemifield was increased (i.e., $75 \%$ compatible-25\% incompatible as compared with $25 \%$ compatible- $75 \%$ incompatible), but no effect of the compatible-incompatible ratio presented in the other hemifield. Because visual stimuli presented to the left or right of a person's fixation point are projected into the contralateral cerebral hemisphere, this finding is consistent with the idea that different degrees of processing selectivity can be adopted by the cerebral hemispheres, with each hemisphere selecting its processing strategy independently of the other hemisphere's "experience" with flanker processing utility. ${ }^{1}$

In order to rule out an interpretation in terms of general location-specific selectivity adjustment (i.e., the adoption of different degrees of processing selectivity for any two locations in the visual field that are associated with differential frequencies of compatible and incompatible stimuli), in Corballis and Gratton's (2003) Experiment 3, target-flanker pairings could occur at a left-sided, rightsided, or central location. Depending on the experimental block, both lateral locations were associated with either mostly (i.e., 75\%) compatible or incompatible trials (i.e., for any experimental phase, flanker utility was the same for stimuli projected to both the left and right hemispheres). In contrast, the central location was associated with a constant $50 \%-50 \%$ ratio throughout the experiment. Despite this invariance, flanker interference for stimuli that were presented at the central location was larger under conditions of predominantly compatible (lateral) trials, thus suggesting that the degree of processing selectivity adopted for the lateral locations was also applied to the central location. Given the confound with visual acuity, however, it is not possible to compare processing selectivity between central and lateral locations by comparing interference effects (in fact, interference at the central location was considerably lower than that at lateral locations, even in blocks with mostly incompatible trials). Thus, although some generalization of selectivity between different locations apparently occurred, the amount of this generalization cannot be determined, and it would clearly be unwarranted to assume that identical degrees of processing selectivity were realized at both the central and the lateral locations. Therefore, location-specific selectivity adjustment independent of hemisphere-specific processing has so far not been ruled out.

Corroborating this view, location-specific compatibility ratio effects that are obviously unrelated to hemispherespecific processing have recently been demonstrated by Crump et al. (2006), who used a modified version of the Stroop task. In that study, participants had to classify the color of rectangles that occurred either above or below the screen center and were preceded by a centrally presented prime color word that could either match (compatible) or mismatch (incompatible) the to-be-named color. Again, different ratios of compatible and incompatible trials (i.e., $25 \%-75 \%$ vs. $75 \%-25 \%$ ) were realized at the two locations, and larger interference occurred at the location at which the proportion of compatible trials was higher. Although the task, the stimuli, and the experimental pro- cedure in the study of Crump et al. differed considerably from those in the study of Corballis and Gratton (2003), the fact that location-specific compatibility ratio effects were observed supports the idea that independent compatibility ratio effects for target-flanker pairings projected to either the left or the right hemisphere may reflect a more general location-specific mechanism of selectivity adaptation. ${ }^{2}$

The goal of the present study was to contrast hemispherespecific and location-specific selectivity adaptation in the flanker task. To this end, we presented stimuli randomly at one of four locations and varied the ratio of compatible and incompatible trials not only between the left and right visual hemifields, but also between different locations within the same hemifield. Because stimuli that occur at locations in the same hemifield are projected into the same cerebral hemisphere, hemisphere-specific adaptation - at least a strong version thereof that assumes that each hemisphere applies the same degree of processing selectivity to all locations within the contralateral hemifield-would predict equivalent flanker interference for such locations. In contrast, location-specific selectivity adaptation predicts different degrees of processing selectivity (and hence different magnitudes of flanker interference) for any two stimulus locations associated with different ratios of compatible and incompatible stimuli, independently of whether they are located in different visual hemifields or in the same visual hemifield.

In the present study, stimuli were presented randomly at a top-left, bottom-left, top-right, or bottom-right location. Ratios of compatible and incompatible trials were varied in a location-specific manner so that in a given phase of the experiment, there was one stimulus location with a probability of .25 for compatible trials and a probability of .75 for incompatible trials (henceforth-focusing on the proportion of compatible trials - 25 location), one stimulus location in the opposite hemifield with a probability of .75 for compatible trials and a probability of .25 for incompatible trials (henceforth, .75 location), and two stimulus locations - one in each hemifield - with probabilities of .50 for both compatible and incompatible trials each (henceforth, .50 locations). We refer to the hemifield that included the .25 location and a .50 location as the $.25-.50$ hemifield and to the hemifield that included the .75 location and a .50 location as the .50-.75 hemifield.

Assuming that each hemisphere selects a single processing strategy on the basis of the combined compatibleincompatible ratios presented at the locations within the contralateral hemifield, one would expect larger flanker interference in the .50-.75 hemifield than in the .25-.50 hemifield. Crucially, however, such a hemispheric account would not be consistent with differential amounts of flanker interference for locations within the same hemifield. In contrast, location-specific selectivity adaptation predicts differential interference effects between locations associated with different compatible-incompatible ratios not only in different but also within the same hemifield. That is, in the .25-.50 hemifield, the .50 location should be associated with larger interference than the .25 location, whereas in the $.50-.75$ hemifield, the .75 location should be associated with larger interference than the .50 location. 


\section{METHOD}

\section{Participants}

One female and 23 male students of the Helmut Schmidt University/University of the Federal Armed Forces, Hamburg participated on a voluntary basis. They ranged in age from 21 to 29 years. All participants were right-handed according to the Edinburgh Inventory (Oldfield, 1971) and had normal or corrected-to-normal vision.

\begin{abstract}
Apparatus and Stimuli
The stimuli were presented on a 17 -in. monitor with a refresh rate of $60 \mathrm{~Hz}$. Participants viewed the screen from a distance of approximately $73 \mathrm{~cm}$. All stimuli were drawn in white on a dark gray background. The letters $\mathrm{H}$ and $\mathrm{S}$ served as targets and flankers. From a viewing distance of $73 \mathrm{~cm}$, the target was presented $2.8^{\circ}$ to either the left or the right of the vertical midline and $2.8^{\circ}$ either above or below the horizontal midline. Targets and flankers extended approximately $0.5^{\circ}$ horizontally and $0.6^{\circ}$ vertically. The flankers were presented in a cross-like fashion around the target (i.e., one above, one below, one to the left, and one to the right of the target). The distance between the inner edges of the target and each of the flankers was $0.3^{\circ}$ horizontally and $0.2^{\circ}$ vertically. Two response keys were used. They were located on an external keyboard and measured $1.0 \times 1.0 \mathrm{~cm}$. One key was located $1.8 \mathrm{~cm}$ to the left and the other $1.8 \mathrm{~cm}$ to the right of the keyboard's saggital midline, which was roughly aligned with the screen center and the participants' midline. For half of the participants, $\mathrm{H}$ was mapped onto the left response key, whereas $\mathrm{S}$ was mapped onto the right response key. This assignment was reversed for the other half of the participants.
\end{abstract}

\section{Procedure}

Each trial began with the presentation of a fixation cross in the center of the screen $\left(0.3^{\circ} \times 0.3^{\circ}\right)$. After a period of $500 \mathrm{msec}$, the fixation cross disappeared, and a target stimulus that was surrounded by four identical flankers was presented randomly at one of the four possible locations for $117 \mathrm{msec}$. Participants were instructed to classify the target letter by pressing the assigned response key as quickly as possible while avoiding errors. In case of a correct response, the next trial started $500 \mathrm{msec}$ after the response with the presentation of the fixation cross. In case of an incorrect response, the German word falsch ("incorrect") occurred for $800 \mathrm{msec}$ slightly below the screen center. Then, the trial was repeated with an identical stimulus. Such repetitions of incorrect trials were not counted as trials and did not enter the statistical analyses.

Participants first received written instructions. After a practice block of 30 trials - in each of which target stimulus, flankers, and stimulus location were chosen randomly (i.e., probability of .50 for compatible and incompatible trials at all four locations) - participants worked through eight experimental blocks of 99 trials each. During an experimental block, the response keys were pressed by the index and middle finger of the same hand. Participants started with their dominant (i.e., right) hand and switched after each experimental block. During a block, the fingers remained on the keys.

The eight experimental blocks were divided in four quarters (i.e., Blocks 1 and 2, Blocks 3 and 4, Blocks 5 and 6, and Blocks 7 and 8). During each quarter, the same location-specific ratios regarding compatible and incompatible trials were maintained. For each quarter of the experiment, a different one of the four stimulus locations was chosen to be associated with probabilities of .25 for compatible trials and .75 for incompatible trials, respectively (i.e., the .25 location). The reverse set of probabilities (i.e., .75 for compatible and .25 for incompatible trials) was always realized at the location diagonally opposite to the .25 location. The two remaining locations (i.e., vertically and horizontally adjacent to the .25 location) were associated with probabilities of .50 for both compatible and incompatible trials. Thus, there were four possible location-specific compatible-incompatible ratio distributions, defined by the .25 location being top left, bottom left, top right, or bottom right. Each of these different ratio distributions was administered in a different quarter of the experiment, and the order of the ratio distributions was counterbalanced across participants (i.e., each participant was administered one of the 24 possible permutations). Despite these constraints regarding the location-specific ratios of compatible and incompatible trials, the stimulus location and the target letter were chosen randomly on each trial, thus resulting in equal expectancy values for targets, responses, flankers, and stimulus locations throughout the experiment.

\section{RESULTS}

The first three trials of each experimental block were considered "warm-up" trials and did not enter the statistical analyses. In addition, data from trials following an erroneous response were discarded (as well as the immediate repetitions of incorrect responses, which were not counted as trials). The reaction time (RT) analysis was confined to correct responses. Also, RT data larger than $1,200 \mathrm{msec}$ were discarded from the analysis $(0.5 \%)$.

ANOVAs with repeated measures on the factors flanker compatible-incompatible, left-right hemifield, $(.25-.50) /(.50-.75)$ hemifield, and within-hemifield lowhigh proportion of compatible trials were conducted on the mean RTs and error proportions. Concerning the fourth factor, in the .25-.50 hemifield, "low" refers to the .25 location and "high" refers to the .50 location, whereas in the $.50-.75$ hemifield, "low" refers to the .50 location and "high" refers to the .75 location.

Concerning RTs, there was a main effect of flanker compatibility $\left[F(1,23)=203.0, M S_{\mathrm{e}}=3045.1, p<.01\right]$, which was modulated by the within-hemifield proportion of compatible trials $\left[F(1,23)=6.5, M S_{\mathrm{e}}=622.6\right.$, $p<.02$ ]. As can be seen in Figure 1, this modulation occurred because in both the $.25-.50$ hemifield and the $.50-.75$ hemifield, flanker interference was higher at the location associated with a higher proportion of compatible trials. Although flanker interference was overall numerically larger in the .50-.75 hemifield than in the .25-.50 hemifield ( 86 vs. $75 \mathrm{msec}$ ), this difference failed to reach significance $\left[F(1,23)=2.5, M S_{\mathrm{e}}=938.3, p=.13\right]$. The main reason for this failure was that interference was almost identical at the two .50 locations $(82 \mathrm{msec}$ in the $.25-.50$ hemifield and $79 \mathrm{msec}$ in the $.50-.75$ hemifield) $[F(1,23)<1]$.

The corresponding ANOVA on error proportions again yielded a main effect of flanker compatibility $[F(1,23)=$ $\left.9.5, M S_{\mathrm{e}}=.05198, p<.01\right]$, indicating increased error proportions on incompatible trials rather than on compatible trials. The overall interference effect was much larger when stimuli were presented in the right than in the left hemifield $(12.2 \%$ vs. $2.1 \%)\left[F(1,23)=12.0, M S_{\mathrm{e}}=\right.$ $.02056, p<.01] .^{3}$ Also, as can be seen in Figure 1, flanker interference was larger in the .50-.75 hemifield than in the $.25-.50$ hemifield $(8.4 \%$ vs. $5.8 \%)[F(1,23)=5.1$, $\left.M S_{\mathrm{e}}=.00321, p<.04\right]$. Although in both the $.25-.50$ and the $.50-.75$ hemifields flanker interference was numerically larger at the location with the higher proportion of compatible trials (see Figure 1), unlike in the RT analysis, this difference was not significant $[F(1,23)<1]$. Interference at the .50 location in the $.25-.50$ hemifield was nu- 


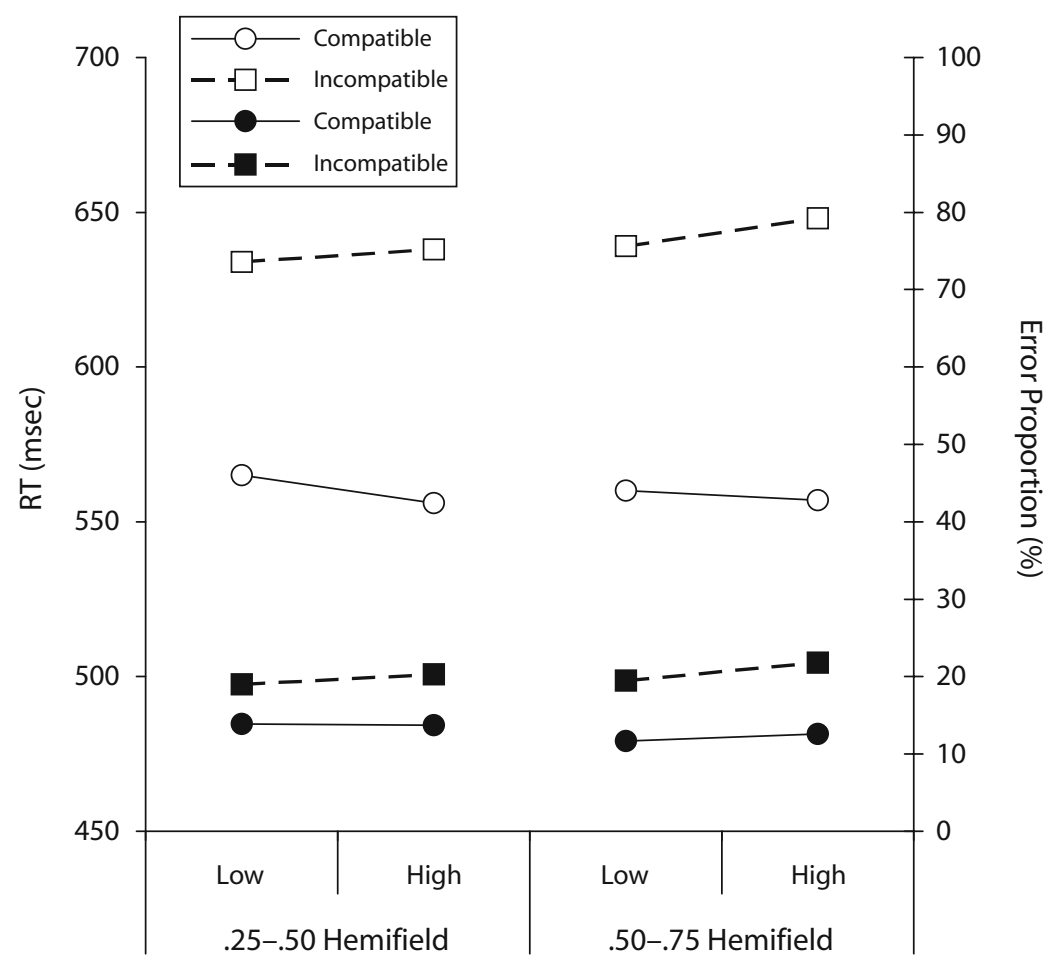

Figure 1. Mean reaction times (RTs, open symbols) and error proportions (filled symbols) as a function of the compatible-incompatible flanking condition and the low-high proportion of compatible trials in both the $.25-.50$ and $.50-.75$ hemifields.

merically lower than that at the .50 location in the $.50-.75$ hemifield $(6.6 \%$ vs. $7.8 \%)$. However, this difference was far from significant $[F(1,23)<1]$.

Although investigating hemisphere-specific processing requires categorizing stimuli according to occurrence in the left versus the right hemifield, given our experimental makeup (i.e., presenting the .25 location and the .75 location diagonally opposite to each other), locationspecific adjustment should also present itself as an interference difference between locations within the same upper or lower hemifield. Therefore, we reanalyzed our data, replacing the left-right hemifield distinction by an upper-lower hemifield distinction. The pattern of results was very similar to that of the original analysis (flanker interference amounted to $69 \mathrm{msec} / 5.0 \%$ at the .25 location, $82 \mathrm{msec} / 7.7 \%$ at the .50 location in the $.25-.50$ upperlower hemifield, $79 \mathrm{msec} / 6.1 \%$ at the .50 location in the $.50-.75$ upper-lower hemifield, and $92 \mathrm{msec} / 7.9 \%$ at the .75 location); however, the interaction between flanker interference and within-hemifield proportion of compatible trials just missed statistical significance $[F(1,23)=4.1$, $M S_{\mathrm{e}}=1024.5, p<.06$, and $F(1,23)=3.5, p<.08, M S_{\mathrm{e}}=$ $.00322, p<.08$, for RTs and errors, respectively].

\section{DISCUSSION}

The experiment of the present study replicated findings of fluctuations of interference elicited by irrelevant flanker stimuli as a function of varying ratios of compatible and incompatible trials, previously accounted for in terms of selectivity adaptation to flanker processing utility (Gratton et al., 1992). Moreover, in line with the results of Corballis and Gratton (2003), marked differences in flanker interference occurred at locations in different visual hemifields that were associated with reversed compatible-incompatible ratios.

More importantly, the present study extended previous research by demonstrating variations of flanker interference regarding stimulus locations within the same hemifield. Specifically, in both the $.25-.50$ and the $.50-.75$ hemifields, flanker interference was higher at the location associated with a larger proportion of compatible trials. Given that stimuli presented in the same visual hemifield are projected into the same cerebral hemisphere, this finding cannot be attributed to hemispheric processing differences, thereby questioning interpretations of between-hemifield interference differences in terms of hemisphere-specific processing. Corroborating a hemisphere-independent, location-specific account, flanker interference was not larger at the .50 location of the $.50-.75$ hemifield than at the .50 location of the $.25-.50$ hemifield. That is, processing selectivity at a .50 location was not influenced by the overall compatibleincompatible ratio administered to the contralateral hemisphere.

Note, however, that although the within-hemifield differences in flanker interference obtained in the present study are inconsistent with a strong hemispheric account 
that assumes that each hemisphere applies the same processing strategy to all locations within the contralateral hemifield, they do not rule out a weaker version in which each hemisphere is assumed to be capable of applying more than one degree of processing selectivity to different locations within the contralateral hemifield. In this view, selectivity at all locations within the same hemifield could still be exclusively controlled by the contralateral hemisphere. Assuming that processing strategies adopted by the same hemisphere are somewhat less independent of each other than are strategies adopted by different hemispheres, evidence for the weaker hemispheric account might be obtained by demonstrating that influences of the compatible-incompatible ratio realized at one location on the interference effect at another location occur predominantly for locations within the same hemifield. (However, no such influence was found in the present study; that is, flanker interference was not larger at the .50 location of the .50-.75 hemifield than at the .50 location of the $.25-.50$ hemifield. As was noted in the Introduction, some generalization between different locations has previously been obtained, and future research is needed to identify necessary and sufficient conditions for its occurrence.)

Although in the present study we adopted a selectivity adjustment interpretation of conflict-frequency effectsthereby following the predominant view in the literature (see, e.g., Botvinick et al., 2001; Corballis \& Gratton, 2003; Gratton et al., 1992) - there are two alternative interpretations that deserve discussion. First, because we did not exert fixation control in our study, it is conceivable that in each quarter of the experiment, participants identified the "hardest" (i.e., .25) location and looked straight at it. Assuming that visual acuity is inversely related to flanker interference, such a strategy could not only account for the interference reduction at the .25 location, but also- considering that in our experimental makeup, the .75 location was always placed diagonally opposite (i.e., most distant) to the .25 location - for the interference maximum obtained at the .75 location. Fixating the .25 location seems an unlikely strategy, however, for two reasons. First, differences in visual acuity should have resulted in a general (i.e., averaged across compatible and incompatible trials) advantage for stimuli viewed more foveally. This is clearly not the case in our data. Second, in a control experiment in which one location was associated with $25 \%$ compatible and $75 \%$ incompatible trials (i.e., the .25 location), and each of the other three locations involved the reversed ratio, flanker interference was again minimal at the .25 location but not enlarged at the diagonally opposite location, in comparison with the other .75 locations.

A second alternative interpretation is based on the possibility of correlational S-R learning in conflict frequency manipulations. ${ }^{4}$ Specifically, given that under conditions of mostly compatible (incompatible) trials flankers cooccur predominantly with compatible (incompatible) responses, associative flanker-response learning may facilitate responding to compatible (incompatible) stimuli, thereby increasing (reducing) interference (Jacoby et al., 2003; see Cohen, Fuchs, Bar-Sela, Brumberg, \& Magen, 1999, and Miller, 1987, for evidence that flankers may gain the power to activate correlated responses, but see Fernandez-Duque \& Knight, in press, Experiment 2B, for a compatibility ratio-dependent modulation of Stroop interference with control of this factor). Note, however, that to apply this interpretation to the results of the present study, one would have to assume associative learning between flanker-location conjunctions and responses. Although we know of no study that precisely addresses to this issue, note that Miller (1987, Experiment 2) failed to obtain evidence for a processing advantage of more frequently presented target-flanker pairings, thereby questioning the idea of associations between stimulus feature conjunctions and responses. Altogether, the results of the present study seem to be best explained by conflict-related, location-specific adjustment of processing selectivity.

\section{AUTHOR NOTE}

This research was funded by Grant KL488/6-2 from the German Research Foundation (Deutsche Forschungsgemeinschaft) to R.H.K. and M.W. The authors thank Bruce Milliken for valuable comments on the manuscript. We also thank Aquiles Luna-Rodriguez for programming the experimental software and Martin Karkour and Dietmar Schröter for collecting the data. Correspondence concerning this article should be addressed to M. Wendt, Helmut-Schmidt-Universität/Universität der Bundeswehr, Institut für Kognitionsforschung, Holstenhofweg 85, D-22043 Hamburg, Germany (e-mail: mike.wendt@hsu-hh.de).

\section{REFERENCES}

Botvinick, M. M., Braver, T. S., Barch, D. M., Carter, C. S., \& Cohen, J. D. (2001). Conflict monitoring and cognitive control. Psychological Review, 108, 624-652.

Cohen, A., Fuchs, A., Bar-Sela, A., Brumberg, Y., \& Magen, H. (1999). Correlational cuing as a function of target complexity and target-flanker similarity. Perception \& Psychophysics, 61, 275-290.

Corballis, P. M., \& Gratton, G. (2003). Independent control of processing strategies for different locations in the visual field. Biological Psychology, 64, 191-209.

Crump, M. J. C., Gong, Z., \& Milliken, B. (2006). The contextspecific proportion congruent Stroop effect: Location as a contextual cue. Psychonomic Bulletin \& Review, 13, 316-321.

Eriksen, B. A., \& ErIKSEN, C. W. (1974). Effects of noise letters upon the identification of a target letter in a nonsearch task. Perception \& Psychophysics, 16, 143-149.

Fernandez-Duque, D., \& KNIGHT, M. B. (in press). Cognitive control: Dynamic, sustained, and voluntary influences. Journal of Experimental Psychology: Human Perception \& Performance.

Gratton, G., Coles, M. G. H., \& Donchin, E. (1992). Optimizing the use of information: Strategic control of activation of responses. Journal of Experimental Psychology: General, 121, 480-506.

Hommel, B. (1994). Spontaneous decay of response code activation. Psychological Research, 56, 261-268.

HüBNER, R. (1997). The effect of spatial frequency on global precedence and hemispheric differences. Perception \& Psychophysics, 59, 187-201.

Jacoby, L. L., Lindsay, D. S., \& Hessels, S. (2003). Item-specific control of automatic processes: Stroop process dissociations. Psychonomic Bulletin \& Review, 10, 638-644.

Logan, G. D., \& ZBrodoff, N. J. (1979). When it helps to be misled: Facilitative effects of increasing the frequency of conflicting stimuli in a Stroop-like task. Memory \& Cognition, 7, 166-174.

Miller, J. (1987). Priming is not necessary for selective-attention failures: Semantic effects of unattended, unprimed letters. Perception \& Psychophysics, 41, 419-434.

OLDFIELD, R. C. (1971). The assessment and analysis of handedness: The Edinburgh Inventory. Neuropsychologia, 9, 97-113.

Stürmer, B., Leuthold, H., Soetens, E., Schröter, H., \& SomMER, W. (2002). Control over location-based response activation in the Simon task: Behavioral and electrophysiological evidence. Jour- 
nal of Experimental Psychology: Human Perception \& Performance, 28,1345-1363.

Tzelgov, J., HeniK, A., \& Berger, J. (1992). Controlling Stroop effects by manipulating expectations for color words. Memory \& Cognition, 20, 727-735.

Yovel, G., Yovel, I., \& Levy, J. (2001). Hemispheric asymmetries for global and local visual perception: Effects of stimulus and task factors. Journal of Experimental Psychology: Human Perception \& Performance, 27, 1369-1385.

Zaidel, E., Clarke, J. M., \& Suyenobu, B. (1990). Hemispheric independence: A paradigm case for cognitive neuroscience. In A. B. Scheibel \& A. F. Wechsler (Eds.), Neurobiology of higher cognitive function (pp. 297-355). New York: Guilford.

\section{NOTES}

1. The assumption of hemispheric independence regarding processing strategies is in line with a widely shared view that the cerebral hemispheres constitute largely independent processors (see, e.g., Zaidel, Clarke, \& Suyenobu, 1990). In fact, studies examining hemispheric specialization often (although not always explicitly) seem to be based on the assumption that the processing of laterally presented stimuli is largely confined to the hemisphere that receives the stimulus - an idea commonly referred to as the direct access model.

2. Extending this reasoning, it is even conceivable that stimulus location is only one out of a large set of different stimulus features that may become associated with distinct processing strategies. Interestingly, however, Crump et al. (2006) observed no context-dependent adaptation effects when they associated different ratios of compatible and incompatible trials with different stimulus shapes (i.e., rectangles vs. circles).

3 . We currently have no explanation for this asymmetry. Note, however, that larger flanker interference for stimuli in the right hemifield is contrary to what one would expect on the basis of findings indicating a specialized role of the right hemisphere to process global stimulus aspects and the left hemisphere to process local stimulus aspects (see, e.g., Hübner, 1997; Yovel, Yovel, \& Levy, 2001).

4. We are grateful to Bruce Milliken for pointing this out.

(Manuscript received December 18, 2006; revision accepted for publication May 6, 2007.) 